\title{
JLAB HIGH-CURRENT CW CRYOMODULES FOR ERL AND FEL APPLICATIONS*
}

\author{
R.A. Rimmer" ${ }^{\#}$ R. Bundy, G. Cheng, G. Ciovati, E.F. Daly, R. Getz, J. Henry, W.R. Hicks, \\ P. Kneisel, S. Manning, R. Manus, K. Smith, M. Stirbet, L. Turlington, L. Vogel, H. Wang, \\ K.M. Wilson, JLAB, Newport News, VA 23606, U.S.A.
}

\begin{abstract}
We describe the activities underway at JLab to develop new CW cryomodules capable of transporting up to Ampere-levels of beam currents for use in ERLs and FELs. Goals include an efficient cell shape, high packing factor for efficient real-estate gradient and very strong HOM damping to push BBU thresholds up by two or more orders of magnitude compared to existing designs. Cavity shape, HOM damping and ancillary components are optimized for this application. Designs are being developed for low-frequency (750 MHz), Ampere-class compact FELs and for high-frequency $(1.5 \mathrm{GHz}), 100 \mathrm{~mA}$ configurations. These designs and concepts can easily be scaled to other frequencies. We present the results of conceptual design studies, simulations and prototype measurements. These modules are being developed for the next generation ERL based high power FELs but may be useful for other applications such as high energy light sources, electron cooling, electron-ion colliders, industrial processing etc.
\end{abstract}

\section{INTRODUCTION}

Proposed high brightness, high energy and high current light sources for research and industry will require a new generation of SRF cryomodules with good HOM damping, high efficiency, good real-estate gradient and moderate or high average power RF couplers. Cost, maintainability and ease of operation will also be factors. Designs for very high current or multi-pass ERL's or high efficiency ERL-FEL's (which impose a very large energy spread on the spent beam), may require low frequency cryomodules $(<\sim 1 \mathrm{GHz})$, with large apertures and high BBU thresholds. For single pass or low to moderate beam currents (up to $\sim 100 \mathrm{~mA}$ ) higher frequency modules in the range of 1.3 to $1.5 \mathrm{GHz}$ are being considered. Actual frequencies will depend on availability of RF sources and compatibility with existing machines and infrastructure. Components and cryomodule conceptual designs are being developed at JLab suitable for both these ranges of applications.

\section{CONCEPTS}

As has previously been reported [1], JLab has developed a $748.5 \mathrm{MHz}$ Ampere-class cryomodule concept aimed at high power compact ERL-based FELs.

*Work supported by Jefferson Science Associates, LLC under U.S. DOE Contract No. DE-AC05-06OR23177, and by The Office of

Naval Research under contract to the Dept. of Energy.

"RARimmer@jlab.org

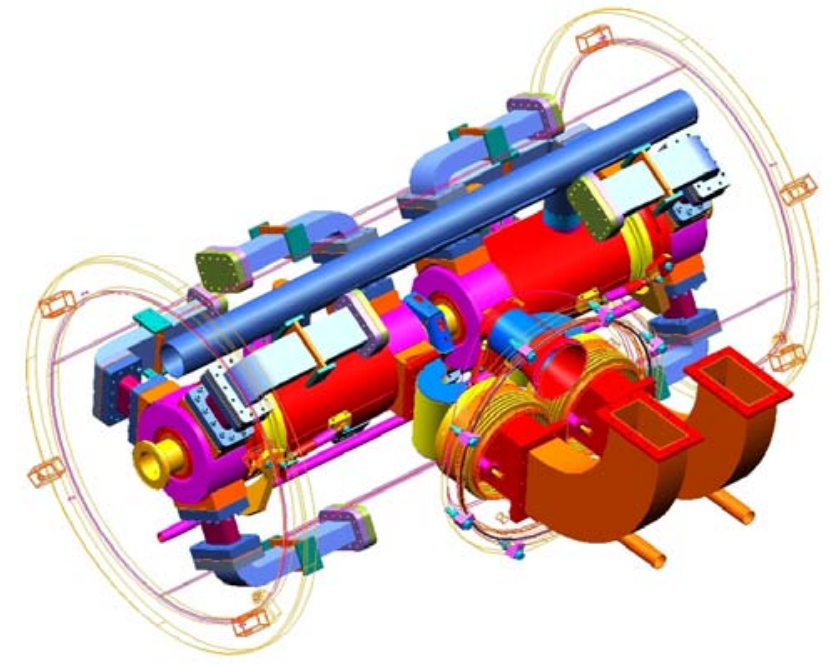

Figure 1. 1.5 GHz high-current cavity pair.

This concept employs multi-cell waveguide damped cavities with room temperature HOM loads and highpower waveguide fundamental power couplers (FPCs). This module could be powered by RF sources in the TV band. The chosen frequency is also a sub-harmonic of the CEBAF RF frequency, $1497 \mathrm{MHz}$, allowing for the possibility of synchronization between machines based on these technologies. As part of the prototyping effort for this project cavities and components have been produced at $1497 \mathrm{MHz}$ to take advantage of existing production and testing infrastructure at JLab. Given the high level of interest in ERL and FEL based light sources in this frequency range we have developed a concept for packaging these designs in an existing CEBAF style cryostat for a cost-effective, usable, proof-of principle module, see figure 1 . For future projects higher packing efficiency could be obtained by using a modified version of the JLab upgrade cryomodule. A machine based on this technology could be synchronized with light sources at $500 \mathrm{MHz}$, or be optimized for any other frequency.

Table 1: JLab high-current cryomodule parameters.

\begin{tabular}{|c|c|c|c|}
\hline & $\begin{array}{c}748.5 \mathrm{MHz} \\
\text { module }\end{array}$ & $\begin{array}{c}\mathbf{1 4 9 7} \mathrm{MHz} \\
\text { module }\end{array}$ & $\begin{array}{c}\mathbf{1 4 9 7} \mathbf{~ M H z} \\
\text { injector }\end{array}$ \\
\hline Voltage & $100-120 \mathrm{MV}$ & $80-100 \mathrm{MV}$ & $10-20 \mathrm{MV}^{*}$ \\
\hline Length & $10.4 \mathrm{~m}$ & $8.5 \mathrm{~m}$ & $2.6 \mathrm{~m}$ \\
\hline \# cavities & 6 & 8 & 2 \\
\hline Aperture & $140 \mathrm{~mm}$ & $70 \mathrm{~mm}$ & $70 \mathrm{~mm}$ \\
\hline $\mathrm{I}_{\max }$ & $1 \mathrm{~A}$ & $100 \mathrm{~mA}$ & $10 \mathrm{~mA}$ \\
\hline HOM Q's & $<10^{4}$ & $<10^{4}$ & $<10^{4}$ \\
\hline RF Power & $0-1 \mathrm{MW}$ & $0-100 \mathrm{~kW}$ & $100 \mathrm{~kW}^{*}$ \\
\hline
\end{tabular}




\section{CAVITY OPTIMIZATION}

The cavity cell shape has been optimized for good RF efficiency, good HOM damping and with HOMs tuned to safe frequencies to minimize HOM power extracted from the beam, which can be a severe problem for high-current machines [2]. HOM damping is provided by waveguide loads on both ends of the cavity, symmetrically placed around the azimuth to avoid steering kicks, figure 2 . The HOM waveguides propagate outwards to room temperature high-power loads and take up very little beam-line real estate. Several prototype cavities have been tested to date including most recently a $748.5 \mathrm{MHz}$ single cell with the high-current profile, see figure 3. All these models have exceeded gradient and Qo specifications with no signs of multipacting [3], figure 4. Preliminary HOM loaded Q's measured on copper singlecell and 5-cell models indicate BBU thresholds greater than 1 Ampere for the low frequency module as predicted by simulations [4]. This should translate to $>100 \mathrm{~mA}$ for the $1497 \mathrm{MHz}$ ERLs although the actual BBU threshold will depend on the specific machine configuration. The 748.5 MHz cavity has five cells, which is long enough to have reasonable packing factor (with the waveguide HOM loads), but short enough to have good HOM damping. The $1497 \mathrm{MHz}$ multi-cell prototypes have also been made with 5 cells but more cells could be used if BBU calculations show they are feasible.

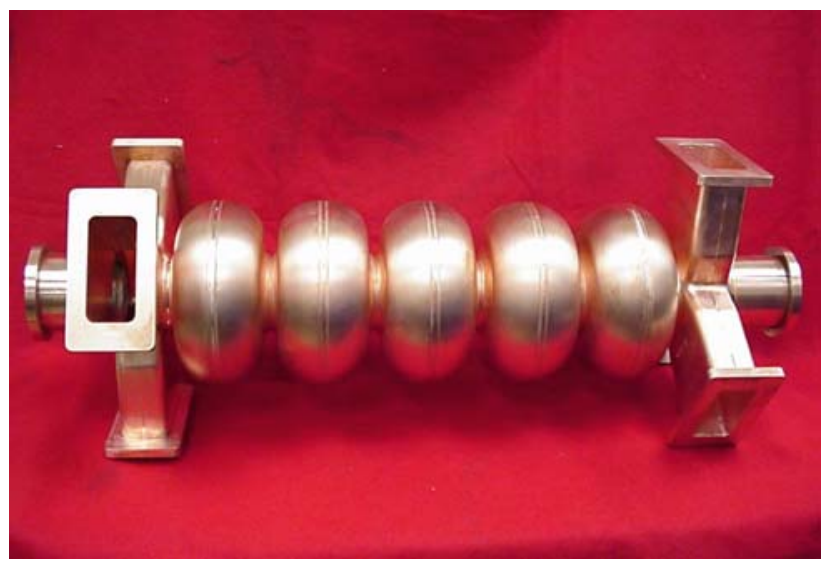

Figure 2. Copper five-cell model of the high-current cavity with waveguide end groups.

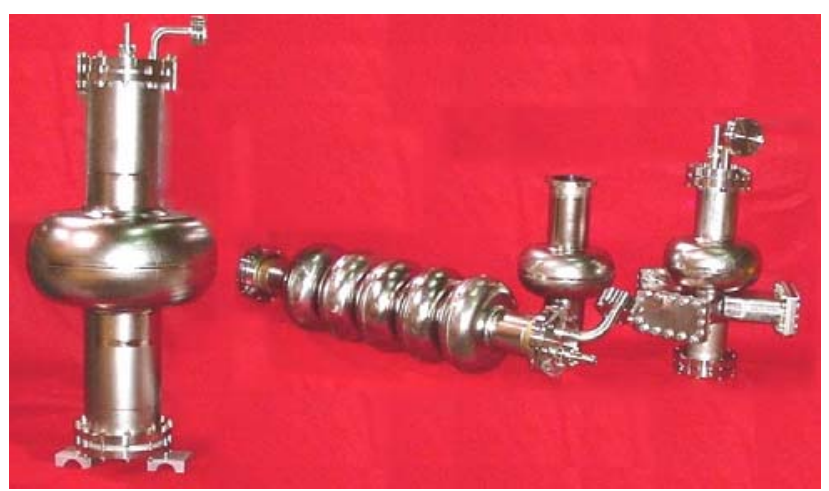

Figure 3. $748.5 \mathrm{MHz}$ and $1497 \mathrm{MHz}$ High-current prototype cavities.

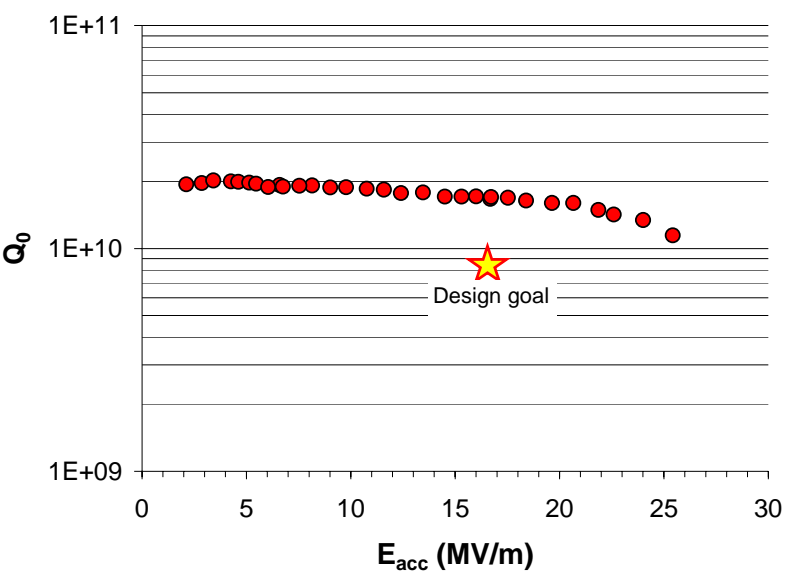

Figure 4: $\mathrm{Q}_{0}$ vs $\mathrm{E}_{\mathrm{acc}}$ for the $750 \mathrm{MHz}$ single cell cavity measured at $2 \mathrm{~K}$.

\section{HOM LOADS}

High power HOM loads with $\mathrm{SiC}$ ceramic tile absorbers capable of dissipating up to $4 \mathrm{~kW}$ each (20 $\mathrm{kW} /$ cavity), with good safety margin have been developed for the Ampere-class module. The broad-band match of this load is shown in figure 5. The loads have also been analyzed using ANSYS multi-physics simulation to take real RF fields and calculate the losses, absorber temperature and thermal stresses in a common model [5], figure 6. A simplified lower power version has been developed for the $1497 \mathrm{MHz}$ cavities using a similar absorber profile but with simplified construction and reduced cooling for the lower current requirements. For currents below $\sim 1 \mathrm{~mA}$ a cryogenic version could be developed, as used in CEBAF, avoiding the static heat leak from the warm waveguides. ANSYS multi-physics analysis was also applied to the cavity HOM waveguide extensions outside of the helium vessel and revealed that superconducting extensions are required for about four more inches $(\sim 100 \mathrm{~mm})$ to mitigate RF losses from the evanescent fields of the cavity fundamental mode (a consequence of the tight coupling of the HOM waveguides to the cavity). These extensions will be cooled by conduction to the helium vessel or by external helium circuits intercepting the outer flanges.

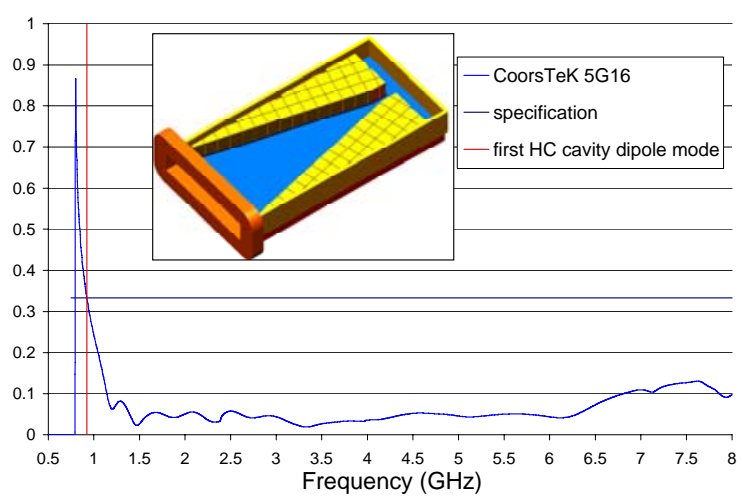

Figure $5 . \mathrm{S}_{11}$ of high-power load using SiC absorber. 


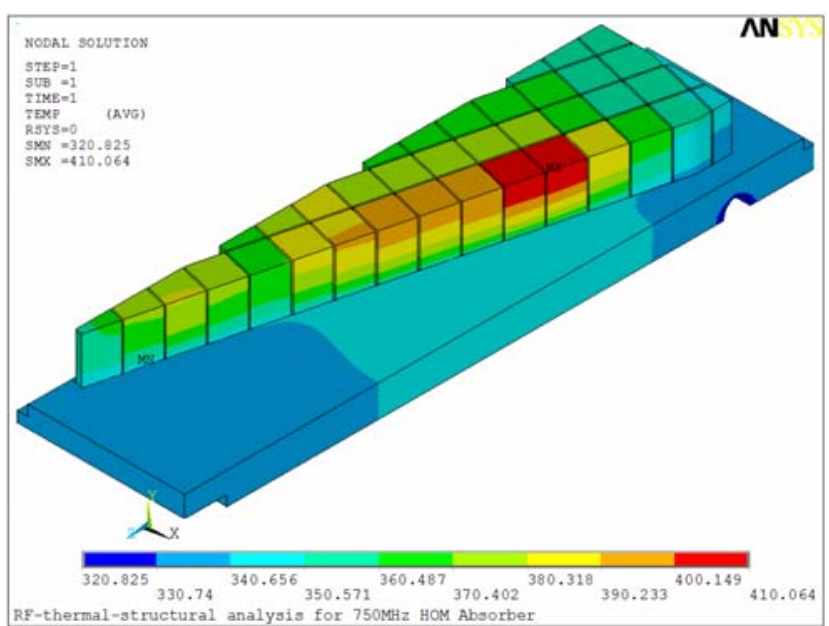

Figure 6: Temperatures $(\mathrm{K})$ in tiles and base plate.

\section{WINDOW AND FPC}

A high power window concept has been developed [6], based closely on the successful B-factory and LEDA windows. This has been prototyped in WR650 for use at $1497 \mathrm{MHz}$ with the cavity prototypes but can be scaled anywhere from 1.3 to $1.5 \mathrm{GHz}$ by simply changing the ceramic thickness. Several fully brazed windows have been fabricated at JLab, figure 7, and will be high-power tested in the near future. One arm of one HOM end group on each cavity does double duty as the fundamental power coupler. By changing the dimensions of the waveguide extension external to the cavity the loaded Q can be changed to suit either the heavily beam-loaded injector or the energy-recovered linac.

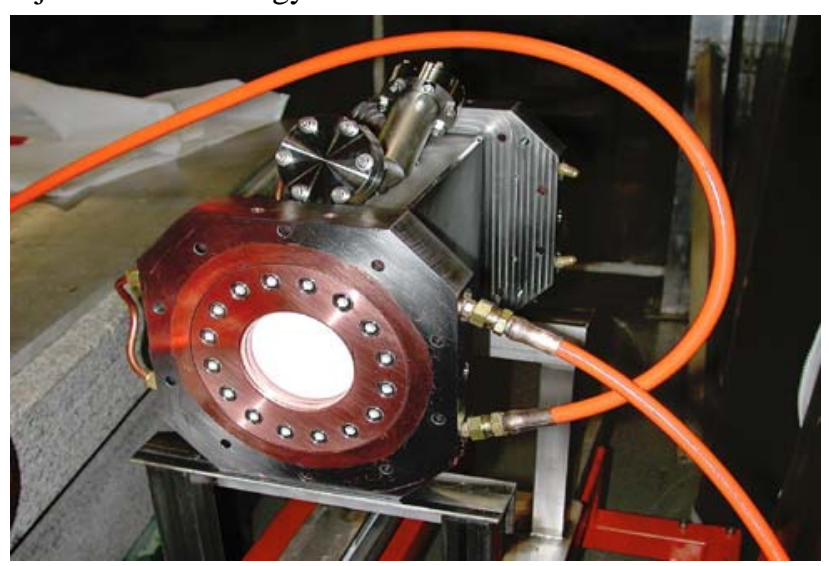

Figure 7. High-power waveguide window test box.

\section{HELIUM VESSEL}

The helium vessel wraps around the HOM waveguides so the whole end group is actively cooled by $2 \mathrm{~K}$ helium, see figure 8, which should ensure stable operation at high gradient. Unlike the original CEBAF module each cavity has its own helium vessel and there are no helium to vacuum flanged joints. The high frequency cryounit concept uses a helium gas return header that interfaces to standard CEBAF end cans. The whole cavity pair with HOM waveguides, tuner, FPC and helium circuits fits in the same volume as the original CEBAF common helium vessel. FPC location and spacing are preserved so that the original CEBAF style vacuum vessel can be used. A new heat shield configuration is required however because the HOM loads are now at room temperature. Cavity tuning is achieved using a "zero insertion length" tuner on the helium vessel so no active length is consumed (figure 8).

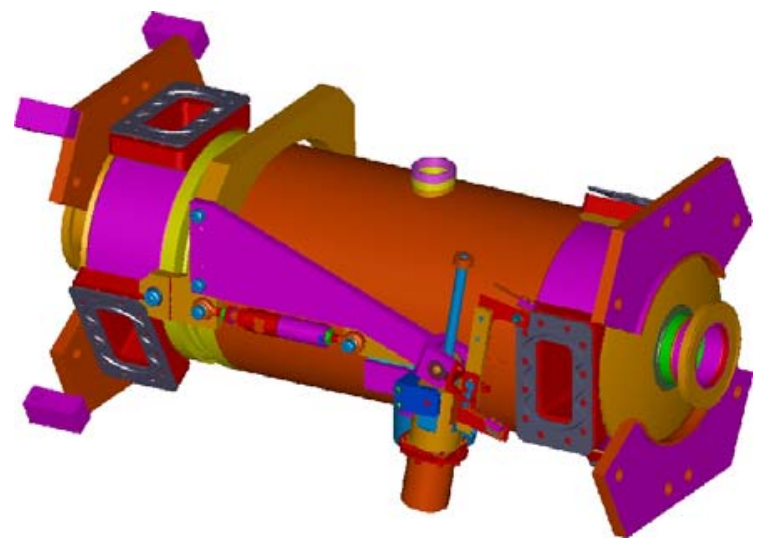

Figure 8. Helium vessel and tuner mechanism.

\section{CONCLUSIONS AND FUTURE PLANS}

We have developed concepts for a low frequency Ampere class cryomodule and a high-frequency $\sim 100 \mathrm{~mA}$ version based on a common design philosophy that yields high real estate gradient, good RF efficiency and is based largely on proven technology. The various cavity prototypes have exceeded goals for gradient and $Q_{0}$ with no signs of multipactor. A final 5-cell $748.5 \mathrm{MHz}$ and two 5-cell $1497 \mathrm{MHz}$ prototypes with full waveguide end groups are nearing completion and will be tested shortly. A proof of principle cryounit with two fully loaded 5-cell $1497 \mathrm{MHz}$ cavities is being developed based on an existing CEBAF vacuum vessel and end cans. This prototype will be completed and tested with beam in the JLab FEL as soon as funding allows. The concepts presented here can easily be scaled to any frequency.

\section{REFERENCES}

[1] R.A. Rimmer et. al., "The JLab Ampere-Class cryomodule conceptual design”, Proc EPAC06, Edinburgh, Scotland.

[2] H. Wang et. al, "Elliptical cavity Shape Optimization for Acceleration and HOM Damping”, Proc. PAC 2005, Knoxville TN.

[3] P. Kneisel, et. al., "Preliminary Results From Prototype Niobium Cavities for the JLab Ampere Class FEL", these proceedings.

[4] H. Wang, et. al., "Simulations and Measurements of a Heavily HOM-Damped Multi-cell SRF Cavity", these proceedings.

[5] G. Cheng, et. al., "RF-Thermal-structural analysis of a waveguide higher order mode absorber", these proceedings.

[6] M. Stirbet et. al., "High-power windows for WR650 waveguide couplers", these proceedings. 